\title{
La lettre de consolation de Plutarque à sa femme traduite par La Boétie et ses prolongements chez Montaigne, Céline et Michaël Fœssel
}

\section{Jean Vignes}

\section{(2) OpenEdition}

Journals

Édition électronique

URL : http://journals.openedition.org/rhetorique/545

DOI : 10.4000/rhetorique.545

ISSN : 2270-6909

Éditeur

UGA Éditions/Université Grenoble Alpes

Édition imprimée

ISBN : 978-2-37747-010-5

\section{Référence électronique}

Jean Vignes, «La lettre de consolation de Plutarque à sa femme traduite par La Boétie et ses prolongements chez Montaigne, Céline et Michaël Fœssel », Exercices de rhétorique [En ligne], 9 | 2017, mis en ligne le 22 juin 2017, consulté le 12 septembre 2020. URL : http://journals.openedition.org/ rhetorique/545; DOI : https://doi.org/10.4000/rhetorique.545

Ce document a été généré automatiquement le 12 septembre 2020.

\section{(7) (-) (2)}

Les contenus de la revue Exercices de rhétorique sont mis à disposition selon les termes de la Licence Creative Commons Attribution - Pas d'Utilisation Commerciale - Partage dans les Mêmes Conditions 4.0 International. 


\title{
La lettre de consolation de Plutarque à sa femme traduite par La Boétie et ses prolongements chez Montaigne, Céline et Michaël Fœssel
}

\author{
Jean Vignes
}

1 D'un point de vue méthodologique, cet article met en œuvre une démarche simple dont on sait la valeur heuristique : la mise en série. Il s'agira de donner à lire (avec un apparat critique minimal) puis de mettre en perspective quatre textes liés entre eux par le fait que chacun est inspiré, très explicitement, de la lecture du précédent, quatre textes qui nous invitent à réfléchir sur le pouvoir de l'écriture face à l'épreuve du deuil.

Voici d'abord un bref aperçu de ce corpus et de la façon dont nous tenterons de l'aborder. Notre point de départ est la «Lettre de consolation de Plutarque à sa femme » écrite à l'occasion de la mort de leur fille Timoxéna, texte que nous lisons dans la traduction française qu'en a donnée La Boétie et que publie Montaigne après la mort de son ami. Lecteur et admirateur de cette belle traduction, Montaigne la dédie à sa propre épouse en 1571 à l'occasion de la mort de leur propre fille Toinette : cette brève dédicace constitue notre deuxième texte, qui a retenu à son tour l'attention de LouisFerdinand Céline. Dans Voyage au bout de la nuit, il met en scène la découverte de cette lettre de Montaigne par Bardamu et en propose à cette occasion une réécriture plaisante et un bref commentaire. Enfin, dans son récent ouvrage Le temps de la consolation, le philosophe contemporain Michaël Fœssel, lecteur de Montaigne et de Céline, propose de leurs textes une analyse comparative que nous aimerions discuter.

On voudrait ainsi montrer que la lettre de Plutarque à sa femme a été à l'origine d'une sorte de débat sur la valeur consolatoire d'une telle lettre, débat auquel ont pris part, sous des formes très différentes, La Boétie, Montaigne, Céline et Fœssel. La question débattue est au cœur de la rhétorique : quel effet une lettre peut-elle produire sur les émotions de celui ou de celle à qui on l'adresse ? Un simple courrier peut-il consoler de la mort d'un enfant? C'est donc ce débat que nous voulons retracer, en l'éclairant par 
une analyse rhétorique des textes de La Boétie et de Montaigne notamment, pour remettre en question la lecture du corpus par Michel Fœssel, qui n'emporte pas notre adhésion.

4 On trouvera donc associés à cet article dans le document intitulé "Trois textes en série : la consolation de Plutarque traduite par La Boétie - Montaigne - Céline », les trois textes qui nous occuperont, précédés chacun d'une brève notice, et ici-même une analyse rhétorique du texte de Plutarque, dans la version annotée qu'en donne La Boétie, analyse suivie d'un commentaire critique de la lecture de Fœssel, sur la base d'une lecture rhétorique plus attentive des textes de Montaigne et de Céline.

\section{Essai d'analyse de la traduction de La Boétie : une rhétorique de la consolation?}

5 On pourrait hésiter d'abord à qualifier d'objet «rhétorique » la lettre de Plutarque à son épouse Timoxéna. Loin de constituer un discours oratoire, marqué par la recherche d'éloquence, le texte relève plutôt du genre de la lettre familière. Même s'il existe bien, à l'époque où écrit Plutarque, une tradition de l'épître de consolation, le genre (epistola consolatoria) paraît moins codifié que d'autres, et laisse beaucoup de liberté dans la disposition. Si rhétorique il y a, c'est donc une rhétorique du cœur ou pectus, guidée moins par le souci de suivre un protocole standardisé (qui en l'occurrence n'existe pas) que par l'expression, à chaud, des affections, c'est-à-dire ici des sentiments suscités chez un père par la mort de son enfant et le chagrin de son épouse. Notre propos est donc moins de repérer les étapes d'une dispositio normalisée que de suivre les mouvements du texte, et ses revirements, qui peuvent paraître erratiques : ils nous semblent figurer, de la façon la plus naturelle qui soit, le conflit entre l'émotion et la raison, mais aussi l'amour d'un mari pour sa femme éloignée. Loin d'une rhétorique envisagée comme artifice, notre lecture privilégiera au contraire l'idée d'un mouvement assez spontané de l'intelligence et du cœur: dans une lettre manifestement écrite sous le coup de l'émotion, et probablement à la hâte, il s'agissait pour Plutarque de tenter de rasséréner son épouse et de se rassurer lui-même en lui témoignant son amour, son estime pour elle et surtout sa confiance : en somme, loin de tancer une faible femme trop encline à se laisser submerger par son chagrin, Plutarque salue au contraire le courage de son épouse, dont il ne parait pas douter une seconde. On peut certes voir là un moyen habile de l'inciter au courage (c'est l'éloge dit parénétique et nous ne négligerons pas cette piste de lecture), mais il n'y a pas lieu d'exclure une interprétation plus simple: la lettre de Plutarque à Timoxéna nous touche parce que c'est une vraie lettre d'amour qui traduit l'admiration profonde qu'inspire cette femme à son philosophe d'époux ${ }^{1}$.

6 Nous laissons aux hellénistes et aux spécialistes de la rhétorique antique l'analyse du texte grec de Plutarque. Nous centrons ici l'attention sur le texte français de La Boétie, et sur le dispositif consolatoire qu'il propose (notamment à l'épouse de Montaigne, mais aussi plus largement à tous les lecteurs et lectrices du temps), dans sa version de 1570 imprimée par les soins de Montaigne.

7 Le texte n'est pas découpé en paragraphes (à une exception près sur laquelle on reviendra), mais se trouve balisé par dix-huit manchettes en italiques qui guident la lecture en attirant l'attention sur les articulations majeures et les passages 
remarquables, non sans souligner la dimension universelle de certains passages, pour en tirer des leçons. Notre lecture s'appuiera, entre autres, sur ces repères (dont on ne peut exclure qu'ils soient l'œuvre de Montaigne lui-même, éditeur du texte en 1571). Afin de faciliter la lecture du commentaire, on mentionnera aussi entre crochets la numérotation traditionnelle des paragraphes de Plutarque.

On observera au passage l'entrecroisement subtil et imprévisible des principaux lieux de la consolation:

- discrète lamentation: dans un premier mouvement de commisération, il s'agit de montrer qu'on comprend la douleur de la perte (amplificatio)

- apaisement (lenitas)

- exhortation au courage et à la vertu (exhortatio), souvent ici sous la forme paradoxale de l'éloge à valeur parénétique.

9 En partie redondant avec le titre (apocryphe), le sous-titre («Plutarque à sa femme, bonne et heureuse vie») est une salutation qui souligne la situation d'énonciation, la destination particulière de la lettre. Nous lisons une missive qui ne nous est pas adressée. Rien n'indique, à ce stade (si ce n'est la dédicace de Montaigne), la dimension potentiellement universelle de cette lettre privée.

[1] L'exorde renforce d'abord ce sentiment d'avoir affaire à quelque chose qui, en quelque sorte, ne nous regarde pas. Plutarque s'attarde d'abord sur les circonstances dans lesquelles, au cours d'un voyage, il a appris la mort de sa fille, puis sur ce qu'il croit savoir de l'enterrement. Mais déjà le commentaire marginal dégage une première leçon à valeur générale ; elle concerne les rites funéraires, mais peut s'appliquer bien plus largement : « Superfluité et vaine superstition à eviter. » De ce premier mouvement, la manchette citée ne retient donc que l'énoncé à valeur parénétique. C'est sur cette recommandation de Plutarque à son épouse que s'appuie la captatio benevolentiae: le philosophe exprime à son épouse sa parfaite confiance; le désir d'édification fait fond sur l'éloge de la destinataire, à laquelle sont prêtées les vertus qu'on cherche à cultiver en elle.

11 [2] S'engage ensuite la commiseratio, l'étape concessive et empathique de la lettre de consolation, celle que Montaigne, dans les Essais, prescrira en ces termes: «il faut ayder d'arrivée et favoriser leur plaincte, et en tesmoigner quelque approbation et excuse. Par cette intelligence vous gaignez credit à passer outre, et, d'une facile et insensible inclination, vous vous coulez aus discours plus fermes et propres à leur guerison.» («De la diversion», III, II, p. 831-832). Plutarque entrelace donc ses premières exhortations («qu'en ceste douleur tu te maintiennes [...] dans les termes du devoir ») et les marques de compréhension. Cette étape procède traditionnellement de l'amplificatio: il s'agit de souligner la grandeur de la perte. Plutarque insiste sur sa propre sensibilité : «je cognois et compren en cest inconvenient ${ }^{2}$, de combien il est grand [...]. Et pour vray, je ne suis ny de bois, ny de pierre : Toy-mesmes, le scais bien ». Le texte souligne la réciprocité de l'empathie (je sais que tu as de bonnes raisons de pleurer; tu sais que je ne suis pas moins sensible; je sais que tu voulais une fille après quatre garçons) et la solidarité des parents dans la douleur: ils ont élevé ensemble leurs enfants. Puis, après l'évocation de cette fratrie nombreuse se dégage, comme gage de l'émotion partagée, le touchant portrait de la défunte.

L'éloge de la petite fille distingue plusieurs arguments justifiant une grande douleur :

- sa naissance longtemps attendue

- le fait qu'elle soit la seule fille de ses parents 
- la «vive affection» qu'elle suscitait. Celle-ci est elle-même justifiée rationnellement par l'énumération des qualités naturelles du caractère de l'enfant: sa gaité (la manchette souligne le «Naturel gay d'une petite fille »), sa façon «franche et naïve », c'est-à-dire naturelle, son bon caractère (« n'ayant rien de cholere et de despit»). L'amplification est renforcée par l'usage de différents procédés d'accumulation: énumération d'épithètes laudatives («Et voyoit on en elle une nature admirable, paisible, douce, et attrempée [modérée]»), polysyndète («Et l'amour qu'elle rendoit à ceux qui l'aymoient, et la recognoissance qu'elle avoit envers ceux qui luy faisoient quelque bien »), rythmes binaires (« donnoit tout à la fois plaisir, et cognoissance d'un naturel humain et débonnaire »).

13 [3] Commence alors, balisé par l'apostrophe "Or ma femme », le long mouvement argumentatif visant à modérer la douleur (lenitas) par des « advertissemens ».

14 L'exemple de Clymène (mis en valeur par une première citation versifiée) illustre l'idée générale selon laquelle "nostre nature fuit volontiers cela dont elle reçoit peine »; d'où le risque que la peine produite par le deuil n'entraîne finalement, par la fuite (ou le refoulement) de cette douleur, une forme d'oubli. L'exhortation, mise en valeur par la manchette "Comment il se faut souvenir des morts", porte donc d'abord sur la nécessité d'entretenir un souvenir positif des défunts. De l'idée de "plaisante memoire ", on glisse ainsi tout naturellement à l'exigence fondamentale que souligne la manchette suivante : "Que le dueil doit estre moderé ».

Plutarque oppose implicitement et très habilement l'attitude joyeuse et gratifiante de la défunte et celle d'un survivant qui cèderait au chagrin : alors que la défunte était louée pour « l'amour qu'elle rendoit à ceux qui l'aymoient, et la recognoissance qu'elle avoit envers ceux qui luy faisoient quelque bien » (fin de [2]), le deuil est assimilé au contraire à une forme d'ingratitude, comme si l'on voulait " pour l'ayse qu'on a receu quelquefois, [...] rendre en payement au double de fascheries et d'ennuys » (fin de [3]).

[4] Par une sorte de mouvement cyclique, Plutarque revient ensuite à son point de départ : il rappelle ce qu'il sait de l'enterrement et loue son épouse pour la modération dont elle a su faire preuve en s'abstenant de tout « appareil somptueux qui sentist sa pompe et superfluité ». On retrouve ainsi le motif fondamental mis en valeur dès la première manchette : "Superfluité [...] à eviter », reformulé ici par une autre maxime à l'infinitif : «La somptuosité ne servir de rien. »

17 Dans la traduction de La Boétie, une habile paronomase souligne l'antithèse entre « la somptuosité » inutile et la "modeste simplicité ». Le constat de la sagesse de son épouse conduit Plutarque à en faire le modèle illustrant une règle plus générale : la modération qu'observe une "femme de bien » qui sait se garder "pure aux festins et aux jeux », il ne convient pas de s'en départir dans le deuil. Certes, comme le souligne la manchette, la "naturelle affection envers les morts » est légitime (exceptionnellement, la manchette souligne ici la concession plutôt que l'exhortation). Mais le «desir desordonné de mener deuil [...] n'est pas moins vicieux à le bien prendre, que la dissolution aux voluptez. " Une série de trois questions rhétoriques, de plus en plus longues, illustre et amplifie cet argument en condamnant l'« Ancienne façon de porter le deuil » (manchette). À la sage discrétion de son épouse, Plutarque oppose l'incohérence de ceux qui fustigent l'abandon aux voluptés mais semblent encourager l'abandon au chagrin par des démonstrations funèbres outrancières. Ce mouvement qui passe de l'éloge au blâme (qui appuie la louange d'une personne sur la critique des autres) va ensuite être exploité une seconde fois. 
18 [5] L'apostrophe "ô ma femme » introduit en effet un nouvel éloge du comportement de l'épouse, suivi de l'évocation de nouveaux repoussoirs. La manchette souligne d'abord "L'honneur que Plutarque fait à sa femme, et pourquoy il la louë tant ». La rhétorique encomiastique fait ici appel à l'hyperbole et à de nombreux procédés de dédoublement comme parallélismes, répétitions ou doublets synonymiques (nous les notons ici par des lettres suivies des chiffres 1 et 2) : «il n'y a un seul des philosophes (a.1) qui nous ont hantez (b.1) et cogneus (b.2), qui ne se soit esmerveillé de l'honneste (c.1) humilité de ton accoustrement, et de la moderation (d.1) et façon reformée (d.2) de ta vie : et n'y a pas un de noz citoyens (a.2), à qui ton honneste (c.2) simplesse aux eglises, aux sacrifices, aux theatres, ne serve d'exemple (e.1) et de miroir (e.2). Et d'autrefois à un pareil besoing qu'à ceste heure, tu fis cognoistre une grande asseurance d'esprit (f.1), et fermeté de cueur (f.2), lorsque tu perdis l'aisné de noz enfants (g.1), et encores depuis alors que nostre beau Charon nous abandonna (g.2). " L'hyperbole culmine dans la conclusion de cet éloge : «Ce sont vrayement les chefs d'œuvre d'un bon cœur et noble, et d'une vive affection. »

19 [6] S'ensuit par opposition le blâme des mauvaises mères, les plus nombreuses, et de leur « deuil sauvage et enragé ». On y retrouvera les mêmes procédés d'amplification.

De cette double confrontation entre le comportement exemplaire de l'épouse et les façons répréhensibles de la plupart des autres parents, Plutarque tire une leçon générale, qui emprunte des formes parénétiques propres à frapper la mémoire. Il allègue d'abord, comme le note la manchette, la «Fable d'Esope touchant le deuil»: un bref apologue où le Deuil fait l'objet d'une personnification. La fable semble supposée connue de l'épouse, car le récit est très elliptique. Mais au-delà de la brève fiction qui le confronte à "Jupiter", le Deuil apparaît comme un personnage envahissant auquel chacun de nous peut avoir affaire. Très habilement, le texte ménage ainsi une profonde continuité entre la fable proprement dite, la peinture des comportements humains et le discours exhortatif qui s'en dégage. Une mise en garde très ferme, et des images qui frappent l'imagination mettent en valeur les précautions à prendre pour éviter l'intrusion du deuil dans l'esprit : « il le faut combattre des l'entree à la porte, et non pas luy quitter le fort, en laissant son habillement, et son poil... » Comme portée par l'allégorisation ésopique du deuil, cette très longue phrase, tableau des conséquences délétères de l'abandon au chagrin, s'amplifie au moyen de nouvelles personnifications : les rites funèbres, si l'on en abuse, «tiennent en serre et diminuent la vigueur de l'esprit, et le mettent en desespoir de trouver issue du mal, et le rendent incapable de consolation, tout obscur et tenebreux ». Puis c'est l'entendement lui-même qui est représenté "enveloppé de ces tristes habits». La suite illustre par une effrayante gradation la déchéance croissante où s'enfonce celui qui laisse le deuil prendre possession de son esprit : on perd d'abord la joie de vivre; on en vient à négliger le soin élémentaire de son propre corps. Une longue maxime comparative (combinant rythmes binaires et ternaires) laisse imaginer les conséquences de cet abandon: «car certes quand le corps est sain et en sa gaillardise, une grand' partie de l'ennuy s'abbat et se relasche, comme le flot à un beau jour quand le temps calme : mais si on laisse le corps rouiller et durcir par le mal gouverner, et qu'il n'envoye plus rien de bon ny de gracieux à l'ame, ains seulement comme des ameres et fascheuses fumees: certes à grand' peine se peult-on ravoir, encores qu'on le vueille. " À cette élégante période, succède une phrase brève qui clôt ce mouvement de généralisation philosophique : « Si grandes sont les passions qui saisissent l'ame ainsi mal menée. » 
21 [7] Un avertissement spécifique met en garde contre les "Visites de folles femmes à craindre » (manchette). Ici encore l'exhortation est encadrée par des éloges : le mari sait par expérience que son épouse n'a pas besoin de tels conseils. Le rappel de l'assistance apportée à la sœur de Theon débouche sur un double paradoxe, dont la pertinence est soulignée en marge ("Exemples bien à propos ») : «Car si on voit bruler la maison de son amy, on esteint la flamme le plus tost que l'on peut, et à la plus grande haste ; et quand on le voit luy mesme se consommer en son esprit et tout enflammé, on luy attise encores le feu! Et certes on n'endure pas, quand quelqu'un a mal aux yeux, qu'il y mette la main, encores qu'il le vueille; et personne ne touche là où son mal luy cuit : et celuy qui est en deuil demeure tousjours assis, se presentant à tous venans expressement, ce semble, pour se faire esmouvoir, et envenimer la playe, à fin que pour un peu de douleur qu'il a, qui le poingt et luy demange, l'ulcere egratigné s'empire tousjours, et devienne plus grand et fascheux. » Si la manchette voit là des exemples, on nommerait plus volontiers anti-similitude cette figure originale: alors qu'il paraîtrait logique et sain que les différents maux évoqués (incendie, mal aux yeux, deuil) fussent traités de la même façon, au contraire l'expérience oblige à constater que le deuil fait l'objet d'un traitement inverse, évidemment nocif. Mais l'application rigoureuse de la similitude serait l'attitude raisonnable qu'il convient d'adopter: c'est la conclusion implicite de la démonstration.

22 [8] Le texte aborde finalement des arguments et des méthodes de consolation proprement philosophiques, qui concernent moins les relations avec autrui, avec l'environnement social, que l'intériorité de la personne endeuillée, les efforts qu'elle peut et doit faire sur elle-même pour juguler sa souffrance.

23 L'injonction suivante propose d'abord une sorte d'exercice mental au service de la consolation («comment la consideration du temps peut servir de consolation»): «Essayes toy en ta pensee de te transferer toy-mesme et remettre à ce temps là, que ceste fille, maintenant morte, n'estoit pas encores née ». Selon Plutarque, la comparaison de ce passé avec le présent oblige à constater que la situation est identique. Il ne faut pas pour autant oublier les deux ans qu'a duré la vie de l'enfant, « mais, comme nous ayant apporté jouissance d'autant de faveur et de bien, les compter pour plaisir ». En effet, « reputer un bien court à grand mal» serait « estre ingrats envers nostre fortune du present qu'elle nous a fait, pour ce qu'elle ne l'a pas augmenté de tant comme nous esperions. »

24 [9] Cette réflexion est suivie, dans l'édition de 1571, d'un alinéa qui met en valeur une nouvelle maxime en marge : "Qu'il se faut contenter de ce qui plait à Dieu.» Plutarque évoquait pour sa part les Dieux, mais La Boétie n'hésite pas à christianiser l'idée stoïcienne de consentement à la nécessité : « Car certainement on ne peut faillir à tirer un bel et plaisant fruict de dire tousjours bien et se contenter de ce que Dieu a voulu, et de prendre à gré, et sans se plaindre, ce que la fortune nous baille. » Il suggère à ce propos un autre exercice mental profitable: cultiver «la souvenance des biens passez", "destourne[r] et retire[r] l'entendement des choses qui le troublent et obscurcissent, pour le remettre en la partie de sa vie qu'il a trouvée la plus belle et la plus claire». C'est pour lui le moyen d'éteindre «entierement sa douleur, ou, pour le moins, l'affoibli[r]et l'amorti[r], la destrempant avecques la meslange de son contraire ». Une nouvelle similitude (soulignée en manchette) étaye cette doctrine par un raisonnement analogique: "Car tout ainsi que les onguents de bonne odeur resjouissent tousjours le sentiment, et si font un preservatif contre les mauvaises 
senteurs : ainsi le pensement du bien receu sert encore de remede necessaire au mal qui survient. » Plutarque est pourtant conscient que de telles similitudes ne valent pas pour tous les hommes : « au moins à celuy qui ne fuit pas la memoire du bien passé, et ne prent pas plaisir d'accuser entierement de tout la fortune ». D'où l'exhortation qui clôt ce mouvement: «de quoy nous nous devons bien garder, et de vouloir calomnieusement blasmer la vie d'entre nous hommes pour quelque tache de malheur, une possible sans plus, qui se trouve en elle, comme en un livre, tout le demeurant estant net et entier. » Plutarque rappelle à sa femme les maximes stoïciennes qu'il a pu prononcer jadis: "Car il te souvient bien de m'avoir souvent ouy dire que les changemens de fortune ne peuvent de guieres esbranler nostre vie, ny avec ses hazards elle ne luy sçauroit faire prendre grand saut. » Il en ajoute une troisième, bien frappée, sur laquelle la manchette attire l'attention ("D'où depend la felicité ») : « toute la felicité ne dépend que d'une bonne et droite resolution, parfaite et accomplie en une habitude ferme et asseuree ${ }^{3}$.» On aura donc noté, dans tout ce paragraphe [9] un très net élargissement du propos. Un logicien parlerait ici de prosyllogisme (on remonte la chaîne syllogisitique). Le thème du deuil de l'enfant s'efface provisoirement dans un raisonnement plus large, à propos de la gestion de la douleur en général.

À ce propos, Plutarque revient ensuite aux rapports avec l'entourage, et aux fâcheuses visites de condoléances auxquelles son épouse est nécessairement confrontée. De même qu'il vaut mieux entretenir le souvenir de ses meilleurs moments, de même, on retiendra de ces visites importunes non les «larmes et plaintes» des visiteurs, mais l'admiration qui les inspire : «ceux là mesmes admirent ton bon heur, à raison des enfants que tu as, et de la grandeur de nostre maison, et de ta vie. » Il suggère même que les visiteurs envient la mère endeuillée: «sans doute, ce seroit une chose merveilleusement desraisonnable qu'il n'y aye celuy de ceux qui te voyent, qui ne print volontiers la condition en quoy tu es, encores avec la charge de l'inconvenient dont toy et moy nous deuillons ».

De fait, selon Plutarque, le sage considère non ce qu'il a perdu mais ce qui lui reste. L'argument s'appuie à nouveau sur deux similitudes: de même qu'il serait sot de s'attarder sur les mauvais vers d'Homère, au lieu d'admirer en son œuvre « tant de belles et grandes inventions, ainsi seroit il de toy, si tu voulois rechercher curieusement les infortunes de ceste vie humaine ». Cette ingratitude serait également comparable à la folie des avares «qui, ayans amassé de l'argent de toutes parts, n'en usent point, [mais se plaignent] quand ils l'ont perdu». Suit un exemple de regret possible aussitôt balayé au moyen de cette méthode positive : «Or, si tu plains ta fille pour estre morte sans avoir esté mariee et porter enfants, tu as de l'autre costé de quoy te resjouir de ce qu'il n'y a aucun de ces biens là qui te défaillent ».

On se tourne ensuite vers des consolations concernant l'enfant disparue. L'argumentation tourne alors à la spéculation métaphysique, autour de la question de la survie de l'âme. Plutarque caresse d'abord l'hypothèse d'une vie après la mort, moins douloureuse que celle d'ici-bas: «si elle est allee en lieu où elle ne sente point la douleur, elle n'a pas besoin qu'on se deuille pour elle». Une question rhétorique fonde la consolation sur notre ignorance de la vie dans l'au-delà : " pour quoy devons nous avoir mal pour raison d'elle, s'il n'y a rien qui luy en face? »

On en revient ensuite, par une sorte de digression, à deux arguments plus psychologiques : d'une part, « la perte des grands biens doit faire cesser le deuil qu'on en pourroit mener, quand par la perte mesme on vient à [...] n'avoir plus besoing des 
choses perdues. » D'autre part, le peu de connaissance et de compréhension des jeunes enfants leur garantit une perte minime en cas de décès. L'affirmation est ici renforcée par une question rhétorique: "Timoxene n'a perdu que peu de bien, de tant qu'elle n'en cognoissoit que bien peu et se rejouissoit de peu. Car comment pourroit on dire qu'elle eust perdu ce dont elle n'avoit pas sentiment et qu'elle ne pouvoit encores ny cognoistre ny comprendre?»

[10] Plutarque revient ensuite à des spéculations métaphysiques. Démentant l'idée qu'après la dissolution du corps il n'y aurait plus ni mal ni tourment («opinion » selon les termes de La Boétie, qui a évidemment partie liée avec l'hypothèse de la disparition de l'âme avec le corps), il souligne que cette doctrine est contraire aux préceptes de la religion grecque et des mystères dionysiaques. Il faut donc " presuppos[er] l'ame estre immortelle » (la manchette, moins prudente, affirme sans hésiter « l'ame estre immortelle ») et l'imaginer comme un oiseau en captivité. Cette analogie accrédite l'hypothèse qu'au contraire l'âme des défunts «ne cesse de s'empescher tousjours des passions et fortunes que nous avons icy ", mais pas dans les mêmes proportions selon l'âge de la mort. Suit en effet un raisonnement complexe fondé sur l'opposition de la vieillesse et du jeune âge. À la question posée en manchette ("Quel mal à reprocher à la vieillesse »), Plutarque répond qu'elle " esloigne l'ame et l'estrange du souvenir de ce qu'elle voyoit au lieu dont elle est venue, et parmy les choses d'icy[-bas] l'appesantit et la rend lourde et grossiere ». À l'inverse, l'âme jeune « est retenue en sa forme naturelle, n'ayant pris du corps qu'une façon de ply encores fraiche et molle». Une nouvelle analogie avec le feu qu'on rallume aisément juste après l'avoir éteint accrédite cette conclusion consolante : l'âme n'a « aucun avantage à reculer » le moment de la mort.

[11] Plutarque tire un dernier argument consolatoire des "coustumes et loix anciennes » qui prescrivent un « Enterrement d'enfants sans solennité » (manchette). Pour lui, cette sobriété s'explique par le fait que les enfants morts en bas âge sont des sortes d'anges : ils «ne tiennent rien de terrien ny des choses terrestres [...] desja estans en un estat meilleur et plus sainct, et au partir d'ici arrivez à une plus belle demeure. »Certes (La Boétie omet de le préciser), tout le monde ne partage pas ces croyances. Mais il semble plus dangereux de les refuser que de les admettre. Le texte se termine donc sur une sorte de maxime à propos des croyances utiles et les lois qu'il convient de respecter: même si l'esprit rationnel peut demeurer sceptique face à certaines croyances, il faut au moins sauver les apparences : «il faut par le dehors en user ainsi comme les loix l'ordonnent, et avoir le dedans encores moins souillé et mieux net, et plus chaste ». Conclusion déjà toute montaignienne ${ }^{4}$ !

31 Mais est-ce bien une conclusion? En l'absence de formule de congé venant clore la lettre, la critique a hésité à considérer comme une péroraison en bonne et due forme ce qui semble plutôt un dernier argument. Au XVI ${ }^{\mathrm{e}}$ siècle, les éditeurs de Plutarque jugent donc le texte inachevé ; c'est le sens de la formule finale: "La fin en est à dire en Plutarque». Nous préférons considérer cette fin un peu abrupte comme un nouvel indice de la liberté de composition de cette lettre. Rhétorique assurément, mais aussi peu codifiée que possible. 


\section{Passages de relais : de La Boétie à Montaigne et de Montaigne à Céline} meurt en 1563 à l'âge de 32 ans, La Boétie n'a rien publié de son vivant ${ }^{5}$. Selon Montaigne,

aiant esté surpris de sa destinée en la fleur de son aage, \& dans le train d'une tresheureuse \& tresvigoureuse santé, il ne pensoit à rien moins qu'à mettre au jour des ouvrages qui deussent tesmoigner à la posterité quel il estoit en cela. Et à l'adventure estoit il assez brave, quand il y eust pensé, pour n'en estre par fort curieux ${ }^{6}$.

Mais «par son testament ${ }^{7}$ ", il a désigné son ami « héritier de sa bibliothèque et de ses papiers ». " Parmy ses brouillars et papiers espars ça et là », Montaigne trouve matière à la composition de deux volumes qu'il publie en 1571, à commencer par celui qui nous intéresse, rassemblant d'élégantes traductions en prose d'inspiration morale: La Mesnagerie de Xenophon. Les Regles de Mariage, de Plutarque. Lettre de consolation de Plutarque à sa femme. Le tout traduict de Grec en françois par feu M. Estienne de La Boétie, Conseiller du Roy en sa Cour de Parlement de Bordeaux, item un Discours sur la mort dudit Seigneur De la Boetie par M. de Montaigne (Paris, Federic Morel, 1571).

choisit de dédier les premiers textes à des conseillers du roi, qui sont auss ses amis: La Mesnagerie de Xenophon à «M. de Lansac, chevalier de l'ordre du Roy, conseiller en son conseil privé, surintendant de ses finances... », Les Regles de Mariage de Plutarque à Henri de Mesmes, conseiller du roi, ami de La Boétie.

étrange concours de circonstances, à la fin août, alors même qu'il est en train de préparer cette publication, Montaigne perd à son tour sa fille unique, Thoinette, âgée de deux mois. En éprouve-t-il alors un grand chagrin? Peut-être pas. Dix ans plus tard, dans le livre I des Essais (1580), il confiera, à propos de ses enfants prématurément disparus : «j'en ay perdu, mais en nourrice, deux ou trois, sinon sans regret, au moins sans fascherie ${ }^{8}$ ». On attribue généralement cette relative équanimité à l'esprit du temps : « une époque habituée à la mortalité infantile ${ }^{9}$ ». Quoi qu'il en soit, en 1571 Montaigne va aussitôt tirer parti de la circonstance : il dédie la Lettre de consolation de Plutarque à sa femme à sa propre épouse, frappée par un deuil comparable à celui de Timoxéna.

Cette brève lettre-dédicace a fait couler beaucoup d'encre. Comme Henri Godard, certains ont pu juger le texte « choquant pour une sensibilité contemporaine, par cette façon de parler d'autre chose, puis de cette mort elle-même, comme si elle ne concernait que la mère, et seulement pour se décharger sur Plutarque de tout effort de consolation $^{10}$ ». D'autres au contraire y voient une "charmante lettre » témoignant d'une " vraie gentillesse matrimoniale ${ }^{11}$ ".

Pourtant, la meilleure analyse de ce texte (en tous cas la moins anachronique) nous semble celle qu'a proposée Paul J. Smith dans son livre Réécrire la Renaissance. Le principal mérite de sa lecture est de se rappeler qu'il ne s'agit pas d'une lettre privée, de ne pas négliger par conséquent la fonction rhétorique et pragmatique de la lettre en tant que dédicace :

Loin de témoigner une quelconque insensibilité comme le pense Bardamu [mais peut-on affirmer que Bardamu le pense ?] il s'agit là plutôt d'une stratégie rhétorique, car le principal objectif visé par la publication est avant tout 
paratextuel : elle sert en effet à introduire la traduction qu'a faite La Boétie de la lettre de Plutarque. Le message contenu implicitement est donc: "La lettre de Plutarque est si bien faite, que moi, Montaigne, j'ai préféré utiliser cette lettre pour consoler ma femme de la mort de notre fille, plutôt qu'en écrire une même ${ }^{12}$.

Pour être plus précis dans l'analyse rhétorique, la lettre de Montaigne se subdivise aisément en trois mouvements.

1) Le premier constitue la captation de bienveillance : en dépit des nouveaux usages, des « reigles de ce temps icy » qu'il récuse (tant sur le plan de la galanterie que sur le plan religieux), Montaigne revendique «la simple façon du vieil aage » dans ses manières avec son épouse : gage de sincérité. Il lui parlera donc de façon directe, «à la vielle Françoise", sans dissimuler sa tendresse. Montaigne prend plaisir à afficher publiquement une grande connivence avec son épouse, dont il fera plus loin sa meilleure amie. Ils se comprennent à demi-mot. Le lecteur étranger que nous sommes est le témoin amusé de cette complicité entre époux, censée garantir la sincérité et la vérité de ce qui suit.

2) Le second mouvement, le cœur du texte, constitue la dédicace proprement dite. C'est d'abord, comme il se doit, la présentation valorisante de l'objet du don: Montaigne fait l'éloge de La Boétie puis des textes que son ami lui a légués à sa mort. Vient ensuite le don proprement dit et sa motivation: de la raison la plus générale ( "Je ne veulx pas chichement en user moy seul, ny ne merite qu'ils ne servent qu'à moy ») on passe à une justification toute particulière, pour souligner l'adaptation du don à la dédicataire, sa pertinence singulière compte tenu des circonstances : «la fortune vous a rendu ce present si propre». Suit la confidence qui donne tout son poids à la dédicace: l'évocation sans détour, mais sans excès de pathos, de la mort toute récente de Thoinette, leur fille.

3) Puis Montaigne s'éclipse presque aussitôt, pour laisser plus vite la place à Plutarque, souverain consolateur. Dérobade aux yeux de certains, qui jugent très sévèrement ce passage, sans bien mesurer qu'il ne s'agit pas réellement d'une lettre privée. Comme le note plus justement P. J. Smith, Montaigne « recourt au topos de la modestie pour faire l'éloge du texte principal [la traduction de La Boétie] en minorant ses propres capacités ${ }^{13} »$. La formule de congé, pour être standardisée, n'en est pas moins significative; nous y reviendrons.

Reste à savoir si Céline, découvrant le texte de Montaigne sans celui de La Boétie, pouvait mesurer les enjeux et le fonctionnement rhétorique du texte de Montaigne dans un dispositif éditorial complexe qu'il n'avait pas sous les yeux. Nous ignorons par quel hasard ou quelle nécessité l'auteur du Voyage découvre la lettre de Montaigne. Mais il la lit très certainement, comme son personnage Bardamu, dans une édition des CEuvres de Montaigne, assurément pas dans celles de La Boétie. Ainsi privée de la Consolation qu'elle introduisait, dont elle n'était en quelque sorte que l'enveloppe, la lettre de Montaigne pouvait lui paraître bien sèche et désinvolte. Qui plus est, Montaigne avait fait dans les Essais (on y reviendra) un vibrant éloge de la traduction de Plutarque par Jacques Amyot ${ }^{14}$, et pouvait passer aux yeux de Céline pour un partisan de ce «style académique » qu'il abhorrait. Il était donc bien tentant de s'emparer de cette lettre pour la tourner en dérision, en faire l'objet d'une géniale parodie.

Celle-ci repose sur toute une série de transformations, à commencer par le remplacement de la petite-fille (dont l'âge n'est plus précisé) par un «fils à eux », qu'on peut supposer plus âgé : pourquoi pas du même âge que Bébert? Ce lien avec la situation tragique du narrateur est aussitôt explicité, avant même la récriture de la 
lettre: "Ça m'intéressait immédiatement ce passage, probablement à cause des rapports que je faisais tout de suite avec Bébert. » modifications sont très nombreuses. La première est l'omission de tout le premier mouvement de la lettre, c'est-à-dire de tout ce qui pouvait suggérer une certaine tendresse du mari pour sa femme. Le second trait saillant est la traduction en langue parlée contemporaine, de registre populaire le plus souvent ${ }^{15}$, avec l'adoption du tutoiement, devenu plus courant entre époux. La syntaxe et la ponctuation sont typiques de Céline : l'époux s'exprime ici exactement comme Bardamu lui-même (d'où l'usage de l'italique par Céline pour distinguer plus aisément le récit du discours rapporté). Les marques d'oralité abondent (interjections, répétitions, clichés: «Ça s'arrangera !... Tout s'arrange dans la vie... Et puis d'ailleurs,... »).

41 Non moins remarquable est l'addition d'un dernier mouvement injonctif sans équivalent chez Montaigne (alors que le retour au vouvoiement peut suggérer une plus grande fidélité au texte original). Certes, la première injonction peut sembler implicite dans le texte source («Prenez-en connaissance ma chère femme! Lisez-la bien!»); mais la suite est pure invention de Céline : «Montrez-la aux amis. Et relisez-la encore!» De même l'affirmation de la tranquillité retrouvée du mari est propre à Céline: "Je suis bien tranquille à présent! Je suis certain qu'elle va vous remettre d'aplomb !... » Rien de tel n'est explicite dans le texte de Montaigne. Seule la signature est authentique, jusque dans la graphie ancienne du possessif, et la reprise ironique de l'adjectif: "Vostre bon mari. Michel.»

Un bref monologue intérieur de Bardamu rapporte ensuite de façon très elliptique ses premières impressions de lecteur: il se dit impressionné («Voilà que je me dis moi, ce qu'on peut appeler du beau travail»), mais par quoi au juste? Puis il imagine (ironiquement?) la réaction de la femme de Montaigne : «Sa femme devait être fière d'avoir un bon mari qui s'en fasse pas comme son Michel. » L'ironie peut suggérer une condamnation implicite du manque d'empathie de Montaigne envers son épouse, perçu comme une sorte d'indifférence égoïste ${ }^{16}$. Mais Céline et/ou Bardamu se ravise(nt) aussitôt : qui sommes-nous pour juger autrui ? Que savons-nous vraiment, à plusieurs siècles d'écart, des sentiments profonds de Françoise et de Michel lui-même? «Enfin, c'était leur affaire à ces gens. On se trompe peut-être toujours quand il s'agit de juger le cœur des autres. Peut-être qu'ils avaient vraiment du chagrin? Du chagrin de l'époque?» La conclusion de ce mouvement est clairement interrogative, et la question n'est nullement «rhétorique ». Elle reste sans réponse. Bardamu (Céline ?) se garde bien de porter la moindre condamnation ${ }^{17}$.

$\mathrm{Au}$ terme de cette première analyse, est-il certain que la parodie de Céline tende à "dévaloriser" la parole de Montaigne comme le suggère Philippe Alméras («En contrefaisant la voix de châtelain de Montaigne, en la transposant dans le registre le plus bas [...], Céline la dévalorise, jusqu'à l'annuler $\left.{ }^{18} »\right)$ ? Peut-on surtout inférer de la récriture de Céline un jugement moral sur l'attitude de Montaigne et a fortiori une remise en question de la confiance que celui-ci accordait à la lettre de Plutarque pour aider son épouse à faire son deuil de leur premier enfant? C'est la question que nous voulons approfondir à la lumière des réflexions du philosophe Michaël Fœssel. 


\section{3. «Malaise dans la consolation »?}

\section{Exercices de rhétorique quand est paru l'ouvrage du philosophe Michaël Fœssel, Le temps} de la consolation, qui cite et commente celui de Montaigne et celui de Céline ${ }^{19}$. Sa lecture, $\mathrm{du}$ fait même de la publicité dont elle a bénéficié grâce à une publication aux éditions du Seuil, suivie d'un entretien sur France-Culture, nous paraît mériter d'être discutée.

On peut encore, écrit-il, lire ces textes pour étudier leur logique et mettre en lumière leurs procédés [...]. Mais un doute subsiste sur la capacité de ces discours à atteindre leur but qui est de triompher d'une tristesse par un savoir. Plus encore que leur dimension moralisante, c'est le lien entre la connaissance et les affects qui apparaît suspect au lecteur contemporain : il est devenu difficile d'admettre que le savoir théorique constitue le remède suprême au malheur ${ }^{20}$.

À l'appui de la thèse d'un «malaise dans la consolation » (p. 139), M. Fœssel présente le texte de Céline comme une « description » de « l'échec de la consolation classique » (p. 140). Or l'analyse qu'il en fait, et qui semble souscrire ou adhérer à ce qu'il croit être la lecture de Montaigne par Céline, repose en fait sur une série d'approximations, voire d'erreurs d'interprétation, qui s'expliquent en grande partie par l'absence de recours aux textes originaux. Nous aimerions reprendre cette lecture de Céline par M. Fœssel, et de Montaigne par Céline, pour souligner certains infléchissements; on évitera ainsi qu'une lecture approximative du texte de Céline n'induise une lecture fausse de la lettre de Montaigne.

premier lieu, M. Fœssel présente la lettre de Montaigne comme " une dédicace en tête d'une édition de Plutarque envoyée par Montaigne à sa femme après la mort de leur fille ${ }^{21} »$. En réalité, il ne s'agit pas d'une édition de Plutarque (en grec), mais bien d'une traduction, éditée par Montaigne lui-même, ce qui change tout: d'une part Françoise de La Chassaigne est supposée lire la consolation de Plutarque en français, soigneusement adaptée dans une langue aisée et accessible; surtout cette traduction est l'œuvre de La Boétie, un ami de la famille, comme on sait ! Céline écrit seulement, et fort plaisamment, que la traduction a été retrouvée «dans des vieux papiers d'un ami à moi »; il ne nomme pas La Boétie mais le lecteur est supposé décoder l'allusion puisque l'amitié de Montaigne et La Boétie est devenue quasi proverbiale à l'époque où écrit Céline $^{22}$. Ainsi le traducteur n'est autre que cet ami parfait que Montaigne présentera dans les Essais comme un autre lui-même, meae partem animae selon le mot d'Horace (Odes, II, xvii) cité dans le fameux chapitre « De l'Amitié ». De l'ami véritable, Montaigne y donnera cette définition : il est « celuy qui n'est pas autre : c'est moy. C'est un assez grand miracle de se doubler ${ }^{23}$ ». Il est impossible de comprendre la lettre de Montaigne sans prendre en compte le prix de cette amitié, et même l'idée que La Boétie pourrait être le meilleur porte-parole de Montaigne.

Fœssel juge ensuite qu'«à part le style [la plaisante familiarité du style parlé de Céline ${ }^{24}$ ] et la référence à l'amour [présente chez Montaigne, omise par Céline, ce qui n'est tout de même pas une différence négligeable], la traduction de Céline est somme toute assez fidèle à l'esprit de Montaigne. Ici et là, on est frappé par l'absence de référence à ce que dit effectivement Plutarque. Le contenu de la parole de l'écrivain grec est absent ${ }^{25}[. .]$.$» . Mais ce raccourci de langage néglige en fait une différence$ fondamentale entre Bardamu et l'épouse de Montaigne. Le personnage de Céline découvre manifestement la dédicace de Montaigne dans une édition récente de ses Euvres, achetée pour un franc chez un bouquiniste, édition bon marché où la lettre 
figure parmi d'autres, en l'absence du texte de La Boétie qu'elle était censée introduire $^{26}$; en somme, il n'a pas sous les yeux la consolation proprement dite, qui ne saurait de ce fait exercer le moindre effet. Au contraire, Françoise de La Chassaigne a en mains à la fois la lettre de son mari et la consolation de Plutarque adaptée en français par un ami de la famille, que son mari considère comme son « double ». Si bien que «l'absence de référence à ce que dit Plutarque» dans le texte de Montaigne n'a rien qui puisse surprendre et moins encore choquer, dans les conditions réelles de diffusion du texte (qu'elle soit domestique ou plus large grâce à l'impression) : la lettre de Montaigne n'est pas censée être publiée isolément, et surtout pas exercer elle-même la consolation. L'interpréter comme un texte isolé, c'est en trahir l'intention profonde, qui n'était que très modestement apéritive. Dès lors que l'on rétablit les conditions originelles de lecture prévues pour la lettre de Montaigne, on est conduit à souligner la confiance qu'il place dans le texte de Plutarque récrit par son ami pour aider son épouse à surmonter son chagrin, pour l'aider mieux que lui-même ne saurait le faire. Et il convient de rappeler que le texte de Céline insiste lui-même sur le fait que Montaigne offre à son épouse cette "lettre" que Bardamu n'a pas sous les yeux, non sans souligner la valeur consolatoire de ce texte, et non de sa propre dédicace :

«Et que je l'ai trouvée si joliment bien tapée sa lettre ma chère femme, que je te l'envoie sa lettre!... C'est une belle lettre! D'ailleurs je ne veux pas t'en priver plus longtemps, tu m'en diras des nouvelles pour ce qui est de guérir ton chagrin !... Ma chère épouse! Je te l'envoie la belle lettre! Elle est un peu là comme lettre celle de Plutarque !... On peut le dire! Elle a pas fini de t'intéresser !... Ah! non! Prenez-en connaissance ma chère femme! Lisez-la bien! Montrez-la aux amis. Et relisez-la encore! Je suis bien tranquille à présent! Je suis certain qu'elle va vous remettre d'aplomb !... »

Pour Fœssel, l'expérience de lecture de Bardamu montre que «Si la médecine ne peut guérir, la littérature s'avère incapable de consoler. Pire, elle semble se réduire à une rhétorique pressée d'en finir avec le malheur des autres ${ }^{27}$." Cette lecture prolonge d'ailleurs celle d'Henri Godard dans son édition de Céline pour la «Bibliothèque de la Pléiade » : pour lui déjà l'épisode illustre "l'impuissance de la littérature en pendant à celle de la science ${ }^{28} »$. Il est pourtant difficile de souscrire à pareille analyse. D'une part, la lettre de Montaigne, surtout privée de la consolation qu'elle est censée introduire, ne saurait représenter la littérature en général ${ }^{29}$ (et il n'y a aucune raison de prêter à Bardamu ou à Céline cette généralisation hâtive). D'autre part, le caractère supposé " pressé » du texte de Montaigne est mal interprété ; Montaigne lui-même ne parle pas de hâte ; c'est Céline qui la lui attribue : «je ne veux pas t'en priver plus longtemps »; or la hâte prêtée à Montaigne est censée (dans le texte de Céline) traduire son enthousiasme pour la lettre de son ami, non son "désir d'en finir avec le malheur des autres ». À moins de supposer que Céline accuse implicitement Montaigne de mauvaise foi dans sa lettre à son épouse (ce que rien n'indique), c'est là un faux procès qui est fait à Montaigne, procès qu'on ne saurait mettre au compte ni de Bardamu, ni de Céline. Enfin, parler de "rhétorique pressée », c'est négliger encore une fois la prose de Plutarque traduite par La Boétie, qui fait partie intégrante du dispositif textuel élaboré par Montaigne pour son épouse (M. Fœssel, qui ne donne pas la référence du texte de La Boétie, ne paraît pas l'avoir consulté).

Certes, M. Fœssel finit par concéder qu'«à la différence du médecin [Bardamu], l'épouse de Montaigne dispose du volume de Plutarque auquel il lui est loisible de se reporter" (p.142). Mais cette concession, très approximative dans sa formulation, débouche sur des conclusions plus contestables encore. En réalité, on l'a vu, Françoise 
de la Chassaigne dispose non du "volume de Plutarque " mais de la traduction de la lettre, publiée immédiatement à la suite de la dédicace de son époux. Non seulement il lui est « loisible » de s'y reporter mais c'est la suite logique de la lecture de la dédicace ; elle n'a qu'à tourner la page. «Son mari n'a pas jugé possible d'assumer pour lui-même cette parole consolante » (p. 142) ? En réalité, c'est exactement l'inverse, et Montaigne l'écrit expressément : «il vous descouvrira mes intentions, et ce qui se peut alleguer en cela beaucoup mieux que je ne ferois moymesmes." En bon héritier de toute la tradition humaniste, et comme il continuera de le faire partout dans les Essais, Montaigne fait confiance aux citations des Anciens pour exprimer mieux que lui-même son sentiment le plus intime. Y voir une sorte de dérobade, un manque d'engagement personnel ou d'empathie, serait méconnaître la vertu de la citation pour un lettré de cette génération, plus largement la dimension universelle prêtée aux grands textes de la tradition classique. "Je ne dis les autres, sinon pour d'autant plus me dire » écrira l'auteur des Essais ${ }^{30}$. C'est très exactement ce qu'il écrit déjà à son épouse ici. Contrairement à ce qu'indique $\mathrm{M}$. Fœssel, Montaigne ne se "reporte » pas à Plutarque comme nous pourrions le faire, il ne l'allègue même pas : il laisse parler la voix de La Boétie paraphrasant Plutarque, et cette voix, c'est la sienne. Il ne saurait mieux dire.

Il n'y a pas lieu non plus de lui reprocher de ne pas « indiquer un passage précis dans le livre» (p. 142). D'une part, en fait de livre, il ne s'agit que d'une lettre de quelques pages (10 feuillets dans l'édition originale, moins de 20.000 caractères, espaces compris), et Montaigne compte bien que son épouse lira l'ensemble de la lettre, au reste inachevée (du moins le pense-t-il), sans qu'il soit besoin d'en extraire des morceaux choisis! Il ne s'agit pas de se plonger dans l'in-folio des Moralia. D'autre part, il faut rappeler que la traduction de La Boétie est assortie de dix-huit manchettes, brèves notes marginales en italique ${ }^{31}$ (peut-être de la main de Montaigne lui-même), qui indiquent avec netteté les principaux arguments et les passages les plus dignes de mémoire; de sorte que les «passages précis » les plus utiles sont clairement indiqués à la diligente lectrice.

51 C'est pourquoi la conclusion de Fœssel quant à la lecture de Plutarque par Montaigne nous surprend : « Pour lui, comme pour Bardamu bien plus tard, le savoir prodigué par l'écrivain grec est enfoui dans l'épaisseur des siècles ${ }^{32}$. Le silence qui accompagne la référence à son contenu indique qu'un tel savoir est devenu inaccessible à un lecteur moderne. » Selon la formule de Valéry, «l'inverse est exactement ce que nous pensons qu'il faut penser ${ }^{33}$ ». Pour Montaigne, comme pour La Boétie, le savoir de Plutarque, ou plus exactement son opinion, et surtout son effort argumentatif pour tenter de juguler le chagrin, de le dépasser, n'ont rien perdu de leur actualité, de leur universalité : chaque parent qui perd un enfant est confronté au même chagrin, et peut tenter par les mêmes raisonnements d'y échapper. Les siècles n'y font rien. Et Céline, lui, l'a bien compris et le suggère avec délicatesse : d'une époque à l'autre, les mots et les usages sociaux peuvent changer, tout comme le style des lettres, mais pas le chagrin, ni probablement le désir de voir ses proches y échapper: "On se trompe peut-être toujours quand il s'agit de juger le cœur des autres. Peut-être qu'ils avaient vraiment du chagrin ? Du chagrin de l'époque ?»

Loin d'être "devenu inaccessible à un lecteur moderne ", le texte de Plutarque, très soigneusement traduit en français par La Boétie, et édité après sa mort par les soins de Montaigne (on voit mal pourquoi Montaigne se donnerait la peine d'éditer ce texte s'il le jugeait inaccessible!) n'est plus «enfoui dans l'épaisseur des siècles ». Tout au 
contraire, il est non seulement mis à la portée de tous les parents qui savent lire, mais il brille d'un nouvel éclat. Montaigne sait évidemment gré à son ami La Boétie d'avoir, l'un des premiers, entrepris ce louable travail de traduction d'un auteur grec difficile et en même temps si utile. Il est tentant d'extraire ici des Essais (II, 4, «A demain les affaires ») l'éloge de Jacques Amyot, le principal traducteur de Plutarque au XVI ${ }^{\mathrm{e}}$ siècle, pour mesurer à la fois l'attachement de Montaigne à son œuvre, sa gratitude envers les bons traducteurs, et la vocation qu'il assigne encore aux traductions pour permettre notamment aux « dames » d'entrer de plain-pied dans les grands textes de l'antiquité :

Je donne avec raison, ce me semble, la palme à Jacques Amiot sur tous nos escrivains François, non seulement pour la naïfveté et pureté du langage, en quoy il surpasse tous autres, ny pour la constance d'un si long travail, ny pour la profondeur de son sçavoir, ayant peu développer si heureusement un autheur si espineux et ferré (car on m'en dira ce qu'on voudra : je n'entens rien au Grec, mais je voy un sens si beau, si bien joint et entretenu par tout en sa traduction que, ou il a certainement entendu l'imagination vraye de l'autheur, ou, ayant par longue conversation planté vivement dans son ame une generale Idée de celle de Plutarque, il ne luy a aumoins rien presté qui le desmente ou qui le desdie); mais sur tout je lui sçay bon gré d'avoir sçeu trier et choisir un livre si digne et si à propos, pour en faire present à son pays. Nous autres ignorans estions perdus, si ce livre ne nous eust relevez du bourbier: sa mercy [grâce à lui], nous osons à cett'heure et parler et escrire ; les dames en regentent les maistres d'escole ; c'est nostre breviaire ${ }^{34}$.

53 À la lumière de telles déclarations (qui sont postérieures), on comprend rétrospectivement que Montaigne se réjouisse dès 1571 (avant la parution en 1572 de la grande traduction des Euvres morales par Amyot) de pouvoir faire profiter ses amis du texte de Plutarque, à commencer par sa propre épouse, qui ne pouvait jusqu'ici le lire ni en grec, ni même en latin.

Si M. Fœssel estime abruptement que « l'auteur des Essais laisse sa femme se débrouiller avec Plutarque ", c'est un raccourci plaisant, qui paraphrase familièrement la phrase de Montaigne : « je laisse à Plutarque la charge de vous consoler ${ }^{35}$ », mais qui ignore tout ce qui précède et tout ce qui suit, qui a pourtant son importance. Résumons-en l'essentiel en soulignant qu'autour de Plutarque, la lettre fait intervenir trois autres actants non négligeables de la consolation : Montaigne lui-même, La Boétie, et Dieu, si bien que le dispositif consolatoire est autrement plus complexe que ne l'imagine M. Fœssel.

En premier lieu, la lettre de notre "bon mary » à son épouse est bien, aussi désinvolte qu'elle puisse nous paraître, une lettre d'amour ou d'amitié, témoignant par sa désinvolture même, par sa familiarité plaisante, d'une forme d'estime et d'attachement profond. D'emblée, il assume publiquement, en dépit de la nouvelle mode qu'il récuse, de "courtiser et caresser encore " sa femme dans sa lettre. Souvenons-nous aussi des confidences de Montaigne sur sa pratique épistolaire, telle qu'il la décrit dans les Essais :

[B] Je hay à mort de sentir au flateur : qui faict que je me jette naturellement à un parler sec, rond et cru qui tire, à qui ne me cognoit d'ailleurs, un peu vers le dedaigneux. [C] J'honnore le plus ceux que j'honnore le moins; et, où mon ame marche d'une grande allegresse, j'oublie les pas de la contenance. [B] Et m'offre maigrement et fierement à ceux à qui je suis. [C] Et me presente moins à qui je me suis le plus donné: [B] il me semble qu'ils le doivent lire en mon cœur, et que l'expression de mes paroles fait tort à ma conception ${ }^{36}$.

Montaigne fait à son épouse la grâce et l'honneur de s'adresser à elle dans ce " parler sec, rond et cru », presque dédaigneux, qui est pour lui le langage de la vérité, tel qu'on 
le doit à ceux qu'on estime, à ceux qu'on aime. Il compte sur sa compréhension à demimot ("vous entendez bien que... »), et n'hésite pas à établir avec elle publiquement une sorte de connivence railleuse («Laissons les dire...»), donnant en spectacle leur complicité, leurs goûts partagés: "Vivons ma femme, vous et moy, à la vielle Françoise.» Plus loin, il la présentera explicitement comme faisant partie de ses « amis »: « je n'en ay, ce croy-je ${ }^{37}$, nul plus privé que vous ». Le chapitre "De l'amitié », au premier livre des Essais, tendra certes à opposer l'amitié et la relation conjugale, mais nous n'en sommes pas encore là en 1570 ; et l'on sait que Montaigne se ravisera encore par la suite : en 1588, il soulignera de nouveau les affinités d'un mariage réussi avec l'amitié («Ung bon mariage, s'il en est, refuse la compaignie et conditions de l'amour. Il tache à representer celles de l'amitié ${ }^{38}$ ») avant d'évoquer les "devoirs de l'amitié maritale ${ }^{39} »$. Quoi qu'il en soit, c'est bien d'une amitié amoureuse que témoigne notre lettre, et Montaigne finit par y lâcher le mot : « vous priant le croire pour l'amour de moy ». La dédicace que nous lisons est un témoignage public d'amour conjugal et réclame en retour (comme autre preuve d'amour) la confiance en la parole consolatoire de Plutarque traduite par La Boétie. On pourrait objecter sans doute que la lettre de Plutarque est plus flatteuse pour l'épouse que celle de Montaigne ; pourtant, le fait de juger la lettre de Plutarque adaptée au cas de sa propre épouse, ne revient-il pas à prêter implicitement à celle-ci les vertus de la première ? Et quel plus beau compliment sous la plume de Montaigne que de la présenter publiquement comme son meilleur ami?

57 Le second point à souligner, c'est justement le rappel de cette amitié fraternelle et « inviolable» pour «feu Monsieur de la Boetie», que Mademoiselle de Montaigne a connu personnellement, et qui est explicitement présenté comme le traducteur. Loin de laisser l'épouse "se débrouiller avec Plutarque ", Montaigne la confie en quelque sorte à son plus cher ami ; ou bien il lui confie son ami (incarné dans son livre, et comme vivant à travers lui), non sans rappeler le prix qu'il attache personnellement à ses travaux littéraires («ses papiers et ses livres, qui m'ont esté depuis le plus favory meuble des miens »). À ce superlatif en hommage à l'œuvre de l'ami disparu répondra surtout le superlatif en hommage à l'épouse amie ( « je n'en ay, ce croy-je, nul plus privé que vous »).

58 Enfin, est-il tout à fait négligeable que la dernière phrase de la lettre soit une prière : «Sur ce, ma femme, je me recommande bien fort à vostre bonne grâce, et prie Dieu qu'il vous maintienne en sa garde " ? Certes, on peut objecter que c'est là une formule convenue pour prendre congé ou clore une lettre, et que Montaigne l'emploie de façon systématique à cette époque, quel que soit le destinataire et l'objet du courrier, si bien qu'on ne saurait prendre une formule aussi standardisée au pied de la... lettre! Mais on peut aussi juger que le choix de cette formule de congé n'est en rien anodin, et qu'il traduit un sentiment profond. On ne sache pas que Montaigne choisisse ses mots à la légère. Mademoiselle de Montaigne n'est plus dès lors abandonnée à Plutarque, mais invitée à surmonter son deuil avec l'aide de Dieu. Cette lecture entrerait en résonance avec la discrète christianisation par La Boétie de la lettre de Plutarque, et avec tout ce qu'on sait par ailleurs du respect par Montaigne des apparences de la catholicité.

On ne saurait donc parler d'une "fraternité dans l'impuissance ${ }^{40}$ entre Céline et Montaigne », ou d'une "solidarité entre les inconsolés ${ }^{41}$ ». Au contraire, comme l'a parfaitement compris Céline, Montaigne ne doute pas de la valeur consolatoire du texte de Plutarque traduit par La Boétie : « tu m'en diras des nouvelles pour ce qui est de guérir ton 
chagrin !... Ma chère épouse! [...] Je suis bien tranquille à présent ! Je suis certain qu'elle va vous remettre d'aplomb !... » Céline lui-même doute peut-être de la puissance consolatrice du texte de Plutarque, mais nullement de la confiance de Montaigne; et c'est plutôt de cette confiance (à ses yeux excessive) qu'il se moque.

On peut du reste souscrire sans réserve à cette affirmation de Michaël Fœssel à propos de Montaigne: «En renvoyant son épouse à une consolation antique, il espère sincèrement que la continuité du temps et des expériences (de Plutarque à elle) lui fournira un motif de réconfort. » (p. 142). On ne saurait mieux dire. Il est seulement dommage, et à vrai dire fort étrange, que ce jugement ô combien pertinent entre en contradiction flagrante avec tout le reste de la démonstration du philosophe, et notamment avec sa conclusion: "Devenue totalement inopérante à l'époque contemporaine, la référence à Plutarque était déjà ambiguë à l'époque où Montaigne écrit : au seuil de la modernité, les grands principes de la consolation antique sont sur le point de disparaître, tout comme a disparu le contenu du texte censé apporter le réconfort au deuil.» (p. 143).

Répétons-le pour conclure : «au seuil de la modernité », dans le livre de La Boétie édité par Montaigne, «le contenu du texte censé apporter le réconfort au deuil» n'a pas disparu; il est au contraire bien présent, magnifié par une traduction nouvelle. Les grands principes de la consolation antique ne sont pas davantage sur le point de disparaître et la sagesse stoïcienne, plus ou moins christianisée, inspirera encore d'innombrables « consolations » au cours des siècles suivants - songeons, pour ne citer qu'un exemple célèbre, à la très fameuse « Consolation à $\mathrm{M}$. Du Périer sur la mort de sa fille » (1598). Quant à « la référence à Plutarque », plus spécifiquement, on sait qu'elle est massive dans les Essais : Montaigne la revendique à plusieurs reprises, et jusqu'à la fin de sa vie (dans la dernière strate des Essais). Il consacre un chapitre spécifique à la «Defence de Seneque et de Plutarque» (II, 32), qui commence par ces mots: «La familiarité que j'ay avec ces personnages icy, et l'assistance qu'ils font à ma vieillesse [C] et à mon livre massonné purement de leurs despouilles, [A] m'oblige à espouser leur honneur. » Plus loin, il souligne à quel point la lecture de Plutarque l'aide à réfléchir et à écrire, au point qu'il ne saurait s'en passer :

Mais je me puis plus malaiséement deffaire de Plutarque. Il est si universel et si plain qu'à toutes occasions, et quelque suject extravagant que vous ayez pris, il s'ingere à vostre besongne et vous tend une main liberale et inespuisable de richesses et d'embellissemens. Il m'en faict despit d'estre si fort exposé au pillage de ceux qui le hantent: $[C]$ je ne le puis si peu racointer que je n'en tire cuisse ou aile ${ }^{42}$.

Cette sagesse de Plutarque est-elle "devenue totalement inopérante à l'époque contemporaine»? M. Fœssel l'affirme sans nuance et semble en effet ne plus y accorder nul crédit. Mais pour que la consolation de Plutarque à sa femme puisse nous toucher, voire contribuer à nous consoler, encore faudrait-il la lire! Rien ne suggère que M. Fœssel ait eu cette chance. En rendant ce texte plus accessible qu'il ne l'était, nous espérons permettre au lecteur de bonne foi de revoir son jugement sur Plutarque, sur la lettre de Montaigne, et même sur la lecture qu'en fait Céline. Car s'il est probable que ces derniers ne partagent pas "une solidarité entre les inconsolés ", il n'est pas plus évident que Céline condamne "la tranquillité d'esprit» de Montaigne. Il la lui envie peut-être, et surtout elle l'interroge. Finalement, le texte de Céline, non dénué d'empathie pour un chagrin possible dont nous ignorons tout, rejoint l'esprit de Montaigne par sa conclusion hésitante et pleine de modestie : que sais-je? 


\section{BIBLIOGRAPHIE}

Alméras Philippe, «Et la chair se fait verbe - coup d'œil sur la bibliothèque de Céline », La Revue des Lettres modernes, Céline 1/4, 1976, p. 123-140.

Amadou Anne-Lisa, « Puissance et impuissance du langage dans le Voyage au bout de la nuit de Céline », Revue romane, $\mathrm{n}^{\circ}$ 14, 1979, p. 5-15.

Aulotte Robert, Amyot et Plutarque. La tradition des Moralia au XVI ${ }^{e}$ siècle, Genève, Droz, 1965. Voir p. 104-105, « La Boétie traducteur de la Consolatio ad uxorem ».

Babut Daniel, « À propos des enfants et d'un ami de Plutarque : essai de solution pour deux énigmes ", Revue des Études Grecques, 1981, vol. 94, n 445, p. 47-62.

Bellosta Marie-Christine, Céline ou l'art de la contradiction, Paris, PUF, 1990 (notamment p. 221).

Feytaud Jacques de, «Céline et le petit Montaigne », Bulletin de la société des amis de Montaigne, 12, 1974, p. 5-28.

Fœssel Michaël, Le temps de la consolation, Paris, Seuil, « L'ordre philosophique », 2015. Voir le chapitre IV, « La parole perdue ».

Lalanne Sophie, «Sur les traces d'une illustre inconnue : Timoxéna, épouse de Plutarque », dans Les femmes dans les sociétés grecques de l'Orient romain (2 juin 2012 - Paris, INHA, Université Paris 1 Panthéon-Sorbonne), actes à paraître.

Leichter-Flack Frédérique, «Intertextualité et exemplarité morale de la littérature : le cas de la souffrance de l'enfant, Dostoïevski / Platonov / Céline », dans E. Bouju, A. Gefen, G. Hautcœur et M. Macé (dir.), L'Exemplarité littéraire. Savoirs de l'exemple, pouvoirs de l'exemplaire, Rennes, PUR, 2007, p. $150-170$.

Plutarque, CEuvres morales, éd. et trad. J. Hani, Paris, les Belles Lettres, 1980, t. VIII.

Smith Paul J., Réécrire la Renaissance, de Marcel Proust à Michel Tournier : exercices de lecture rapprochée, Amsterdam, Rodopi, 2009, p. 76-87, « Présence de Montaigne dans le Voyage au bout de la nuit ».

\section{NOTES}

1. Notre analyse rejoint ici, par un tout autre biais, celle de P. Schmitt-Pantel et V. SebillotteCuchet dans leur article récent, «Mères et politique dans les Histoires d'Hérodote et dans les Vies et les Moralia de Plutarque : pistes de recherche ", Cahiers Mondes anciens (consultable en ligne sur Revues.org), $\mathrm{n}^{\circ} 6,2015, \S 33-34$ : «Timoxéna, l'épouse de Plutarque, est [...] louée à plusieurs reprises pour des traits de caractère et des qualités morales qui lui sont propres et ne sont pas liés à la maternité : sa simplicité, sa constance devant le malheur, son refus de se laisser aller à une manifestation excessive de la douleur. [...] Elle apparaît davantage comme un individu partageant les attitudes morales et les croyances de son époux, et ayant de tout temps fait preuve d'un caractère plein de sagesse et de retenue. Vis-à-vis de la petite fille morte, les deux parents sont à égalité. [...] La mère est avant tout une femme - ici son épouse - qui a une stature morale et un comportement indépendant de son rôle de mère. La lecture des Préceptes de mariage [également traduits par La Boétie] donne la même impression. Ils ne contiennent aucun conseil concernant la maternité et la paternité, ce qui est le cœur du traité est l'amour conjugal. » 
2. Inconvenient : malheur.

3. En marge : D'où depend la felicité.

4. Montaigne écrit par exemple : « le sage doit au dedans retirer son ame de la presse, et la tenir en liberté et puissance de juger librement des choses ; mais, quant au dehors, qu'il doit suivre entierement les façons et formes receues. » (Essais, I, 23, « De la coustume et de ne changer aisément une loy receue ", éd. P. Villey, Paris, PUF, "Quadrige», 1965, p. 118). Toutes les références aux Essais renvoient à cette édition.

5. Sauf un poème liminaire de 44 vers latins à la mémoire de son ami défunt Jules César Scaliger, en tête des Scaligeri Poetices libri septem de 1561.

6. Lettre de Montaigne à M. de Foix (1571), CEuvres complètes, éd. M. Rat, Paris, Gallimard, «La Pléiade », 1962, p. 1368. Voir aussi la lettre de Montaigne à Michel de L'Hospital.

7. «Advertissement au lecteur» (Euvres complètes, op. cit., p. 1719).

8. Fascherie : " chagrin profond », selon P. Villey, éditeur de Montaigne. À propos de ses six filles, il confiera aussi : "J'ay deu la pareille [une éducation sans violence] aux enfans que j'ay eu ; ils meurent tous en nourisse; mais $[\mathrm{C}]$ Leonor, $[\mathrm{B}]$, une seule fille qui est eschappée à cette infortune, a attaint six ans et plus [...]» (Essais, II, 8, p. 389).

9. H. Godard, notice citée, p. 1247.

10. H. Godard, notice citée, p. 1247. D’une façon générale la critique célinienne tend à pérenniser ce jugement comme s'il faisait autorité. Certains accentuent encore la condamnation de l'attitude de Montaigne et postulent qu'elle est le fait de Céline ; Frédérique Leichter-Flack, par exemple, analyse « la traduction célinienne et tout ce qu'elle dénonce : désinvolture et indifférence du père (comme si le deuil de l'enfant ne concernait que la mère, et encore par un excès de sensibilité féminine), facilité indécente avec laquelle le mari se décharge de la responsabilité de consoler son épouse endeuillée ». La critique évoque ensuite son " effroi » à la lecture du texte originel de la lettre de Montaigne, "presque aussi choquant que la traduction célinienne.» («Intertextualité et exemplarité morale de la littérature », op.cit., p. 124).

11. J. Lacouture, Montaigne à cheval, Paris, Seuil, 1996, p. 149.

12. P.J.Smith, Réécrire la Renaissance, de Marcel Proust à Michel Tournier:exercices de lecture rapprochée, Amsterdam, Rodopi, 2009, p. 76-87, « Présence de Montaigne dans le Voyage au bout de la nuit » (citation p. 82).

13. Ibid.

14. Le même Jacques Amyot que Céline prendra pour cible dans son fameux article « Rabelais, il a raté son coup» (Réponse de L. F. Céline à Robert Poulet dans Le Meilleur Livre du Mois, 1957) : « Non. C'est pas lui qui a gagné. C'est Amyot, le traducteur de Plutarque : il a eu, dans les siècles qui suivirent, beaucoup plus de succès que Rabelais. C'est sur lui, sur sa langue, qu'on vit encore aujourd'hui. Rabelais avait voulu faire passer la langue parlée dans la langue écrite : un échec. Tandis qu'Amyot, les gens maintenant veulent toujours et encore de l'Amyot, du style académique. Ça c'est écrire de la m... : du langage figé. Les colonnes d'un grand quotidien du matin, qui se flatte d'avoir des rédacteurs qui écrivent bien, en est plein. Ça donne un cloaque à verbe bien filé, à phrases bien conduites, avec, à la fin de l'article, une petite astuce innocente. Pas dangereuse, pas trop forte, pour ne pas effrayer le public. C'est ça l'échec de Rabelais, c'est ça l'héritage d'Amyot. De la vraie m..., je continue.

Rabelais a vraiment voulu une langue extraordinaire et riche. Mais les autres, tous, ils l'ont émasculée, cette langue, jusqu'à la rendre toute plate. Ainsi aujourd'hui écrire bien, c'est écrire comme Amyot, mais ça, c'est jamais qu'une "langue de traduction". Une de nos contemporaines presque célèbre a dit une fois en lisant un livre: "Ah! que c'est beau à lire, on dirait une traduction!" voilà qui donne le ton. C'est ça la rage moderne du français : faire et lire des traductions, parler comme dans les traductions. » 
15. Comme souvent chez Céline, le registre stylistique est en fait variable : au moins un passage relève d'un style plus châtié, moins oral : "une certaine lettre que Plutarque envoyait lui aussi à sa femme dans des circonstances tout à fait pareilles aux nôtres ».

16. À vrai dire, la critique célinienne se divise lorsqu'elle tente de préciser ce que dénonce le passage. Selon Christine Bellosta, Céline dénoncerait «la vaine prétention du stoïcisme à nous aider à supporter l'insupportable, l'inefficacité de la sagesse face aux épreuves du réel » (Céline ou l'art de la contradiction, Paris, PUF, 1990, p. 221). Selon Frédérique Leichter-Flack, il s'agirait de disqualifier moralement «la tranquillité d'esprit que ce recours [à Plutarque] apporte à Montaigne" (op.cit., p. 125) ; on verra que M. Fœssel doute pour sa part de la confiance de Montaigne en Plutarque... si bien que Céline, loin de condamner Montaigne, le rejoindrait dans l'intranquillité...

17. À cet égard, notre lecture est beaucoup moins catégorique que celle de Frédérique LeichterFlack qui entend Bardamu « régler définitivement son compte avec Montaigne en conclusion de sa lecture ». Parlant de Céline, elle évoque encore « la sévérité de son jugement sur Montaigne ». Voir son article « Intertextualité et exemplarité morale », op. cit., p. 124-126.

18. Ph. Alméras, «Et la chair se fait verbe. Coup d'œil sur la bibliothèque de Céline », La Revue des Lettres modernes, 1, 4, 1976, p. 124.

19. M. Fœssel, Le temps de la consolation, Paris, Seuil, 2015, chap.4, "La parole perdue ", p. 139-143.

20. Ibid., p. 139. L'insistance sur l'idée de «savoir » est elle-même étrange dans ce contexte. Ni Plutarque, ni Montaigne ne prétendent dispenser un "savoir théorique»; il s'agit plus modestement de partager une réflexion personnelle inspirée par l'expérience.

21. Ibid., p. 141, suit une citation de la fin de la lettre de Montaigne. Cette première erreur, dont découle tout le reste, témoigne d'une information de seconde main : M. Fœssel s'inspire ici et ailleurs d'un article traduit du norvégien, qu'il ne cite pas, mais dont il tire l'essentiel de sa réflexion sur le texte de Montaigne (A.-L. Amadou, «Puissance et impuissance du langage dans le Voyage au bout de la nuit de Céline ", Revue romane, $\left.\mathrm{n}^{\circ} 14,1979\right)$. On y lit notamment : " cette lettre constituait la dédicace d'une édition de Plutarque que Montaigne avait trouvée parmi les papiers d'un ami défunt et qu'il avait envoyée à sa femme après la mort de leur fille. » (p. 13-14). Amadou ignore déjà qu'il s'agit d'une traduction de Plutarque et que l'ami de Montaigne est La Boétie.

22. Cf. par exemple Georges Brassens : «C'étaient pas des amis de luxe [...]. C'étaient pas des amis choisis / Par Montaigne et La Boétie, / Sur le ventre ils se tapaient fort, / Les copains d'abord. »

23. Essais, I, 28, p. 193.

24. À vrai dire, la lettre de Montaigne à sa femme témoigne aussi de la familiarité qui sied entre époux. On y reviendra plus loin. Voir aussi sur ce point J. de Feytaud, «Céline et le petit Montaigne ", Bulletin de la Société des Amis de Montaigne (BSAM), 12, 1974, p. 5-28.

25. Fœssel, op. cit., p. 141. Ici encore, la comparaison suit pas à pas l'analyse d'A.-L. Amadou (op. cit., p. 13) : «L'essentiel de la lettre - inviter la mère en deuil à se reporter à Plutarque, sans toutefois indiquer en quoi consistent les paroles consolatrices de l'écrivain grec - est néanmoins fidèlement reproduit et même abondamment paraphrasé. Et ce qui est surtout paraphrasé, c'est cette absence d'indications sur le contenu des paroles de Plutarque. Le texte ne donne que des renvois, des assurances variées quant à l'effet bienfaisant de la lettre de Plutarque. »

26. Comme dans l'édition M. Rat des CEuvres complètes (Paris, Gallimard, «Pléiade », 1962), édition qu'utilise M. Fœssel et à laquelle il renvoie (op. cit., p. 141, n. 3).

27. Ibid., p. 141.

28. H. Godard, notice du Voyage au bout de la nuit, op. cit., t. I, p. 1248.

29. La source de cette idée est encore une fois l'article cité d'A.-L. Amadou : « voici donc Bardamu devant la possibilité de trouver un refuge dans la littérature."

30. Montaigne écrit ailleurs : " La parole est moitié à celui qui parle, moitié à celui qui l'écoute. » (III, 13, p. 1088). Comme l'écrit Antoine Compagnon (Un été avec Montaigne, Paris, Éd. des 
Équateurs, 2013, p. 82-83) : « suivant la complémentarité du moi et de l'autre dont Montaigne fait souvent l'éloge, la parole, à condition d'être une parole vraie, est partagée entre les interlocuteurs, et l'autre parle à travers moi. »

31. Voir les notes de notre édition, où ces marginalia sont reproduites.

32. Encore un clin d'œil tacite à A.-L. Amadou: "Incapable de consoler la mère en deuil, Montaigne ne peut que la renvoyer à Plutarque, et ce que dit celui-ci se cache dans la nuit des siècles ».

33. Variétés, III, «Question de Poésie », dans Paul Valéry, Euvres, éd. J. Hytier, Paris, Gallimard, « Bibliothèque de la Pléiade », t. 1, 1957, p. 1292.

34. Montaigne, op. cit., II, 4, « A demain les affaires », p. 363-364.

35. Henri Godard semble reprocher aussi à Montaigne de «se décharger sur Plutarque de tout effort de consolation » (op. cit., t. I, p. 1248).

36. Essais, I, 40, « Considération sur Cicéron », p. 253.

37. La modalisation nous semble renforcer l'affirmation. Elle traduit non l'hésitation ou le doute mais l'intime conviction.

38. Ibid., III, 5, « Sur des vers de Virgile », p. 851.

39. Ibid., III, 9, « De la vanité », p. 975.

40. Encore une idée et une formule de M. Fœssel empruntées, littéralement et sans la citer, à A.L. Amadou: «l'embarras du philosophe peut être confondu avec celui de Bardamu: le texte de Montaigne peut être adapté au style propre du récit. Son message - ou absence de message produit un effet qui n'est pas seulement parodique (bien qu'il soit parodique aussi), mais qui confère à ce petit texte du "bon mari Michel" une voix dans l'ensemble des voix qui, chez Céline, expriment la fraternité dans l'impuissance ".

41. Fœssel, op. cit., p. 143.

42. Essais, III, 5, « Sur des vers de Virgile », p. 875.

\section{AUTEUR}

\section{JEAN VIGNES}

Université Diderot - Paris 7, CERILAC 